\title{
High TBX2 expression predicts poor prognosis in non-small cell lung cancer
}

\author{
Z. ZHANG, Y. GUO* \\ Department of Respiratory Medicine, First Affiliated Hospital of Xinxiang Medical College, Weihui 453100, China \\ *Correspondence: yuepengguo@163.com
}

Received December 19, 2013 / Accepted January 16, 2014

\begin{abstract}
Deregulated expression of T-box2 (TBX2) has been implicated in malignancies. However, the expression pattern and the role of TBX2 in non-small cell lung cancer (NSCLC) remain unclear. The aim of this study was to explore the clinical value of TBX2 in patients with NSCLC. We examined TBX2 expression in 40 NSCLC tissues and matched adjacent noncancerous tissues by quantitative RT-PCR (qRT-PCR) and Western blotting. Furthermore, the TBX2 protein expression was analyzed by immunohistochemistry in 416 NSCLC samples. Kaplan-Meier method and multivariate Cox regression model were used to evaluate the prognostic value of TBX2 expression in NSCLC for disease survival. The expression of TBX2 was significantly elevated in NSCLC tissues as compared with adjacent non-cancerous tissues $(\mathrm{P}<0.001)$. The high expression of TBX2 in NSCLC was significantly correlated with lymph node metastasis and tumor node metastasis (TNM) stage. Kaplan-Meier survival analysis revealed that high expression of TBX2 correlated with poor prognosis of NSCLC patients $(\mathrm{P}<0.01)$. Multivariate analysis showed that TBX2 expression was an independent prognostic marker for overall survival of NSCLC patients $(\mathrm{HR}=2.21,95 \% \mathrm{CI}=1.349-3.905, \mathrm{P}=0.0015)$. Our results suggest that high $\mathrm{TBX} 2$ expression is associated with poor prognosis of NSCLC patients, and TBX2 may be a prognostic biomarker for NSCLC.
\end{abstract}

Key words: TBX2, non-small cell lung cancer, prognosis, biomarker

Lung cancer is one of the deadliest cancers worldwide, with the highest mortality among all cancers [1]. Non-small cell lung cancer (NSCLC) accounts for $80 \%$ of primary lung cancers. The prognosis of NSCLC is poor and the 5-year survival rate of this type of lung cancer is not up to $20 \%$ [2]. Chemotherapy is the standard first-line chemotherapy for advanced NSCLC. However, drug resistance and metastasis remain a major factor influencing the clinical outcome of patients [3]. The identification of new marker predicting the prognosis is important for to improve the early diagnosis and prognosis of patients with NSCLC [4].

T-box2 (TBX2) is a member of the T-box family of transcription factors, which plays a critical role in embryonic development [5]. Recent studies indicate that TBX2 has been implicated in cell cycle regulation and cancer [6]. TBX2 gene is deregulated in breast cancer, melanoma and pancreatic cancer [7-10]. However, we have known very little about its expression and role in NSCLC. In the present study, we investigated the expression of TBX2 in NSCLC by qRT-PCR, Western blot and immunohistochemistry and then analyzed the correlations between TBX2 expression and its clinicopathological parameters. In the end, we verified that TBX2 was upregulated in NSCLC in comparison with the corresponding normal mucosa. Importantly, in our study, we reported first that TBX2 was an independent prognostic factor for NSCLC.

\section{Patients and methods}

Patients. We conducted this retrospective study using a database of all patients $(n=416)$ who underwent curativeintent surgical resection of a primary tumor at Department of Respiratory Medicine, First Affiliated Hospital of Xinxiang Medical College between February 2003 and February 2008 was analyzed. Information on clinicopathologic characteristics and surgical approach was collected based on clinical and histopathologic reports. Outcome data, including perioperative mortality and long-term survival, was recorded. None of the patients received either radiotherapy or chemotherapy before tumor resection. Patients with disease recurrence or metastases were treated with platinum-based systemic chemotherapy. This study was approved by the First Affiliated Hospital of Xinxiang Medical College Ethics Committee, 
China and written informed consent was obtained from all patients.

Quantitative RT-PCR (qRT-PCR). Total RNA was extracted using TRIzol reagent (Invitrogen, USA) from 40 fresh cancerous and control tissues according to the protocol. An equal amount of RNA $(8 \mu \mathrm{g})$ was reversely transcribed into cDNA by Reverse Transcriptase (Invitrogen, USA) according to the manufacturer's instructions. TBX2 and GAPDH were then amplified by quantitative real-time PCR using the following primers: TBX2: forward: 5'-AGTGGATGGCTAAGCCTG3', reverse:5'-ACGGGTTGTTGTCGATC-3'; GAPDH: forward: 5'-CTCCTCCTGTTCGACAGTCAGC-3', reverse: 5'-CCCAATACGACC AAATCCG-3'. Gene amplification was performed in an ABI 7900HT real-time PCR system using SYBR Green master mix (Invitrogen, USA). The mixture was preheated for $10 \mathrm{~min}$ at $95^{\circ} \mathrm{C}$ and followed by 38 cycles of amplification $\left(30 \mathrm{~s}\right.$ at $95^{\circ} \mathrm{C}$ and $1 \mathrm{~min}$ at $59^{\circ} \mathrm{C}$, respectively). The CT value of each sample was calculated, and the relative expression of TBX2 mRNA was normalized to the GAPDH (Ct method).

Western blot analysis. The 40 freshly frozen NSCLC or non-cancerous tissue samples were homogenated in a RIPA buffer (Qiagen, China). After centrifugation at 12,000 rpm, $4^{\circ} \mathrm{C}$ for $20 \mathrm{~min}, 60 \mu \mathrm{g}$ of protein samples was run on a $12 \%$ SDS-PAGE gel and transferred to polyvinylidene difluoride membrane (Millipore). After blocking non-specific binding sites for 60 min with $5 \%$ non-fat milk, the membranes were incubated with rabbit monoclonal antibody against TBX2 (1:1,000; Santa Cruz), and GAPDH (1:1,000;Santa Cruz) at $4^{\circ} \mathrm{C}$ over night, respectively. Then, membranes were washed with TBST for three times, 15 min each time and then incubated with HRP-conjugated anti-rabbit secondary antibody (1:5,000; Santa Cruz) for $60 \mathrm{~min}$ at room temperature. The membrane was developed by an enhanced chemiluminescence system (ECL; Millipore) after washed with TBST for three times. The intensity of the protein bands was determined by densitometry using Image J software.

Immunohistochemistry. Paraffinic-embedded tissues from 416 NSCLC patients were subjected to immunohistochemistry analysis. The slides were immersed in EDTA (pH 8.0) and boiled for $20 \mathrm{~min}$ in the microwave oven for the antigen retrieval. After rinse with PBS, endogenous peroxidase was blocked with $0.3 \%$ hydrogen peroxide for $20 \mathrm{~min}$ at room temperature. The slides were incubated with the anti-TBX2 (1:50; LifeSpan BioSciences, Inc.) in a humidified chamber at $4^{\circ} \mathrm{C}$ overnight. Following additional wash with PBS for three times, the sections were sequentially incubated with horseradish peroxidase-conjugated secondary antibody (LifeSpan BioSciences, Inc.) at $37^{\circ} \mathrm{C}$ for $30 \mathrm{~min}$ and then washed three times with PBS. Finally, diaminobenzidine tetrahydrochloride (DAB) was used for the signal development. PBS was used as negative control.

The total TBX2 immunostaining scores were calculated as the sum of percent positivity of stained tumor cells and the staining intensity. TBX2 showed predominantly nuclear stain- ing. Immunostaining was recorded as predominantly nuclear. The percent positivity was scored as " 0 " $(<5 \%$, negative), " 1 " (5-25\%), "2" (25-50\%), “3” (50-75\%), "4" (>75\%). The staining intensity was scored as "0" (no staining), "1" (weak staining), " 2 "(moderate staining), and " 3 " (strong staining). Both percent positivity of cells and staining intensity were determined under double-blind conditions. The TBX2 immunostaining score was calculated with the value of percent positivity score plus staining intensity score, ranged from 0 to 12 . TBX2 expression level was defined as following: ' - '(score 0-3), ' + ' (score 4-6), ' ++ ' (score 7-9), and +++ ' (score $\geq 10$ ). Based on the TBX2 expression levels, the NSCLC patients were divided into two groups: low TBX2 expression (- and + ) and high TBX2 expression $(++$ and +++$)$.

Statistical analysis. Levels of TBX2 are expressed as mean \pm SEM. The non-parametrics Kruskal-Wallis test was used to analyze the correlation between the TBX2 levels with clinicopathologic characteristics. Spearman correlation analysis was used to examine the relationship between continuous variables. Kaplan-Meier method and the log-rank test was performed to analyze the survival. Multivariate analysis was conducted to determine an independent impact on survival using the Cox proportional hazard method. $\mathrm{P}<0.01$ was considered statistically significant. Statistical analyses were conducted using the SPSS 16.0.

\section{Results}

TBX2 mRNA and protein expression were elevated in NSCLC tissues. To test the TBX2 protein expression level, we used Western blot to detect 40 fresh surgical NSCLC cancerous samples and the paired adjacent non-cancerous tissues from the same patients. As a result, the TBX2 protein expression level was significantly higher in cancerous tissues than that in corresponding adjacent non-cancerous tissues (Figure 1A-B, $\mathrm{P}<0.001)$. All the 40 paired tissues were tested in triplicate. To support the results of the Western blot analysis, we explore the protein expression of TBX 2 by qRT-PCR on the same 40 paired NSCLC cancerous tissues and their adjacent non-cancerous tissues. Consistently, the mRNA expression of TBX2 expression was markedly elevated in NSCLC cancerous tissues in comparison with their corresponding non-cancerous tissues $(\mathrm{P}<0.001$, Figure $1 \mathrm{C})$.

Relationship of TBX2 expression with clinicopathological variables in NSCLC. Immunohistochemical staining showed that TBX2 was expressed mainly in the nucleus. Immunostaining was recorded as predominantly nuclear (Figure 2). In NSCLC tissues, the high expression rate of TBX2 was $66 \%$ (276 of 416), significantly higher than that in adjacent noncancerous lung tissues $(9 \%, 36 / 416)(\mathrm{P}<0.001)$. The correlations between TBX2 expression and clinicopathologic features are shown in Table 1. The high expression of TBX2 in NSCLC was significantly correlated with lymph node metastasis $(\mathrm{p}=0.021)$ and tumor node metastasis $(\mathrm{TNM})$ stage $(\mathrm{p}=0.0031)$. 

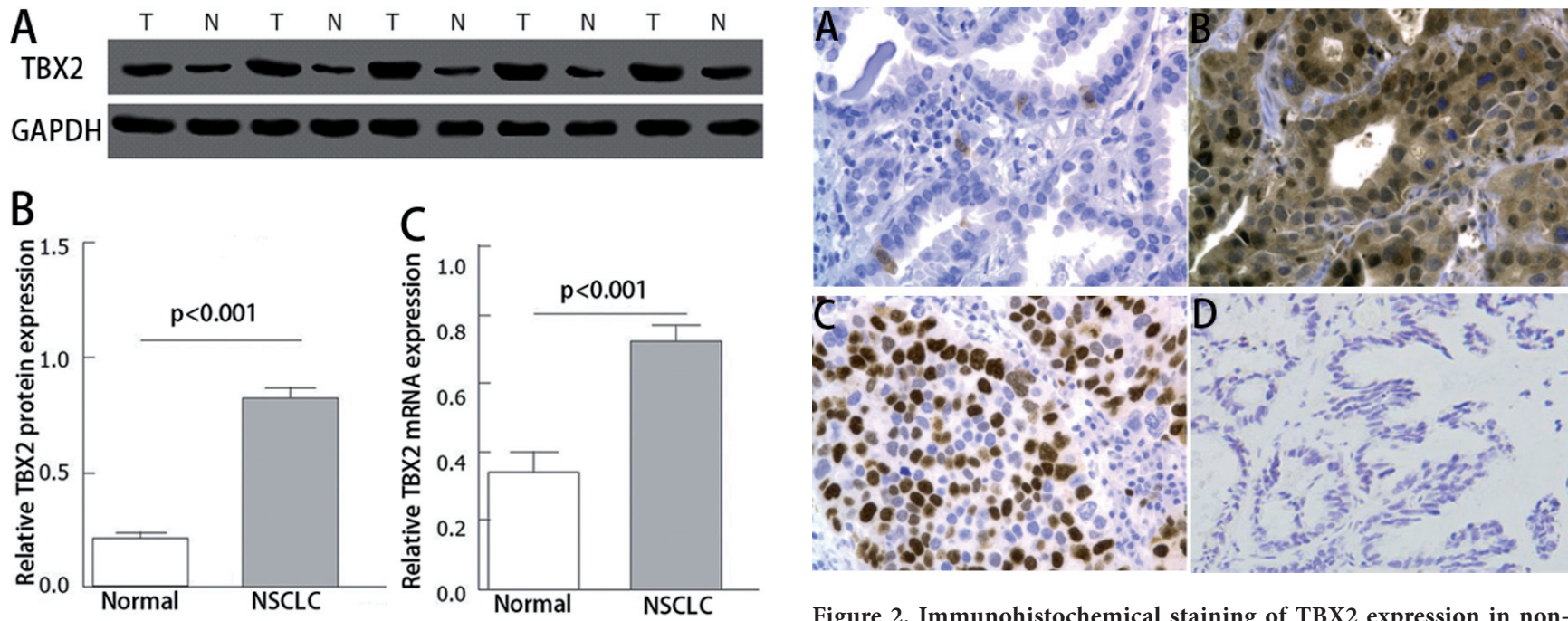

Figure 1. Expression of TBX2 in non-small cell lung cancer (NSCLC) tissues and paired adjacent non-cancerous tissues by Western blotting and qRTPCR. A. Representative Western blotting images of TBX2 protein expression in Cancerous tissues and the matched adjacent Non-cancerous tissues from 5 NSCLC patients. B. Relative Expression of TBX2 in 40 non-small cell lung cancer (NSCLC) tissues and the matched adjacent noncancerous tissues. C. Relative mRNA expression of TBX2 in 40 non-small cell lung cancer (NSCLC) tissues compared to matched adjacent non-cancerous tissues as assessed by qRT-PCR.

High TBX2 expression correlates with poor prognosis. Kaplan-Meier survival curves demonstrated that patients with high TBX2 expression exhibited impaired overall survival compared with those with low TBX2 expression tumors ( $\mathrm{P}=0.007$, Figure 3). Univariate analysis demonstrated that high TBX2 expression was a significant prognostic factor for decreased survival $(\mathrm{P}<0.001)$ (Table 2). The multivariate Cox proportional hazards model revealed that high TBX2 expression was a significant independent factor for poor overall survival $(\mathrm{HR}=2.21,95 \% \mathrm{CI}=1.349-3.905, \mathrm{P}=0.0015$, Table 2$)$.

\section{Discussion}

In the recent years, deregulation of TBX2 has been observed in some types of human cancers, and it remains unclear whether the TBX2 deregulation is also an event in human NSCLC. To explore the role of TBX2 in NSCLC, we investigated the expression patterns of TBX2 in human NSCLC tissues and the correlation between TBX2 expression levels and the clinicopathologic features of NSCLC. Our current study indicates that the expression of TBX2 was significantly elevated in NSCLC tissues as compared with adjacent noncancerous tissues. Multivariate analysis showed that TBX2 expression was an independent prognostic indicator for overall survival of NSCLC patients. These results suggest that high TBX2 expression may act as an independent biomarker for poor prognosis in NSCLC.

Figure 2. Immunohistochemical staining of TBX2 expression in nonsmall cell lung cancer (NSCLC) tissues and normal matched adjacent noncancerous tissues. TBX2 staining was observed mainly in the nucleus of NSCLC cells. TBX2 protein expression was obviously higher in cancer tissues compared with that in normal mucosa. A Score 1: staining of TBX2 expression, B Score 2: staining of TBX2 expression, C. Score 3: staining of TBX2 expression, D. Score 0: negative staining of TBX2 expression in normal adjacent non-cancerous tissues.

Table 1. Association of TBX2 expression status with clinicopathologic features in 416 non-small-cell lung cancer (NSCLC) patients

\begin{tabular}{|c|c|c|c|c|}
\hline NSCLC & $\mathrm{n}$ & $\begin{array}{c}\text { Low } \\
\text { expression }\end{array}$ & $\begin{array}{c}\text { High } \\
\text { expression }\end{array}$ & $\mathrm{P}$ \\
\hline Total & 416 & 140 & 276 & \\
\hline Gender & & & & 0.397 \\
\hline male & 288 & $104(36.1)$ & $184(63.9)$ & \\
\hline female & 128 & $36(28.1)$ & $92(71.9)$ & \\
\hline Age at diagnosis (years) & & & & 0.562 \\
\hline$\leq 60$ & 164 & $36(22)$ & $128(78)$ & \\
\hline$>60$ & 252 & $104(41.3)$ & $148(58.7)$ & \\
\hline Tumor diameter $(\mathrm{cm})$ & & & & 0.635 \\
\hline$\leq 3$ & 140 & $40(28.6)$ & $100(71.4)$ & \\
\hline$>3$ & 276 & $100(36.2)$ & $176(63.8)$ & \\
\hline Histological type & & & & 0.886 \\
\hline Squamous cell & 188 & $64(34)$ & $124(66)$ & \\
\hline Adenocarcinoma & 220 & $76(34.5)$ & $144(65.5)$ & \\
\hline Others & 8 & 0 & $8(100 \%)$ & \\
\hline Differentiation & & & & 0.540 \\
\hline Well & 32 & $12(37.5)$ & $20(62.5)$ & \\
\hline Moderate & 264 & $96(36.4)$ & $168(63.6)$ & \\
\hline Poor & 120 & $32(26.7)$ & $88(73.3)$ & \\
\hline Lymph node metastasis & & & & 0.021 \\
\hline No metastasis & 212 & $116(54.7)$ & $96(45.3)$ & \\
\hline Metastasis & 204 & $24(11.8)$ & $180(88.2)$ & \\
\hline Stage with TNM & & & & 0.0031 \\
\hline Stage I & 200 & $104(52)$ & $96(48)$ & \\
\hline Stage II & 112 & $20(17.9)$ & $92(82.1)$ & \\
\hline Stage III & 104 & $16(15.4)$ & 88 (84.6) & \\
\hline
\end{tabular}


Table 2 Univariate and multivariate analysis of overall survival in non-small-cell lung cancer (NSCLC) patients

\begin{tabular}{|c|c|c|c|c|c|c|}
\hline \multirow[b]{2}{*}{ Parameters } & \multicolumn{3}{|c|}{ Univariate analysis } & \multicolumn{3}{|c|}{ Multivariate analysis } \\
\hline & $\mathrm{HR}$ & $95 \% \mathrm{CI}$ & $\mathrm{P}$ & $\mathrm{HR}$ & $95 \%$ CI & $\mathrm{P}$ \\
\hline \multicolumn{7}{|l|}{ Gender } \\
\hline Male vs. female & 1.042 & $0.790-1.384$ & 0.871 & & & \\
\hline \multicolumn{7}{|l|}{ Age (years) } \\
\hline$<56$ vs. $\geq 56$ & 0.945 & $0.726-1.301$ & 0.910 & & & \\
\hline \multicolumn{7}{|l|}{ Smoking status } \\
\hline Never smoker vs smoker & 1.010 & $0.702-1.496$ & 0.473 & & & \\
\hline \multicolumn{7}{|l|}{ T classification } \\
\hline T1-T2 vs. T3-T4 & 0.956 & $0.832-1.099$ & 0.529 & & & \\
\hline \multicolumn{7}{|l|}{ C classification } \\
\hline I-II vs. III & 0.961 & $0.818-1.129$ & 0.629 & & & \\
\hline \multicolumn{7}{|l|}{ TBX2 expression } \\
\hline positive and negative & 2.882 & $1.597-4.218$ & $<0.0001$ & 2.21 & $1.349-3.905$ & 0.0015 \\
\hline
\end{tabular}

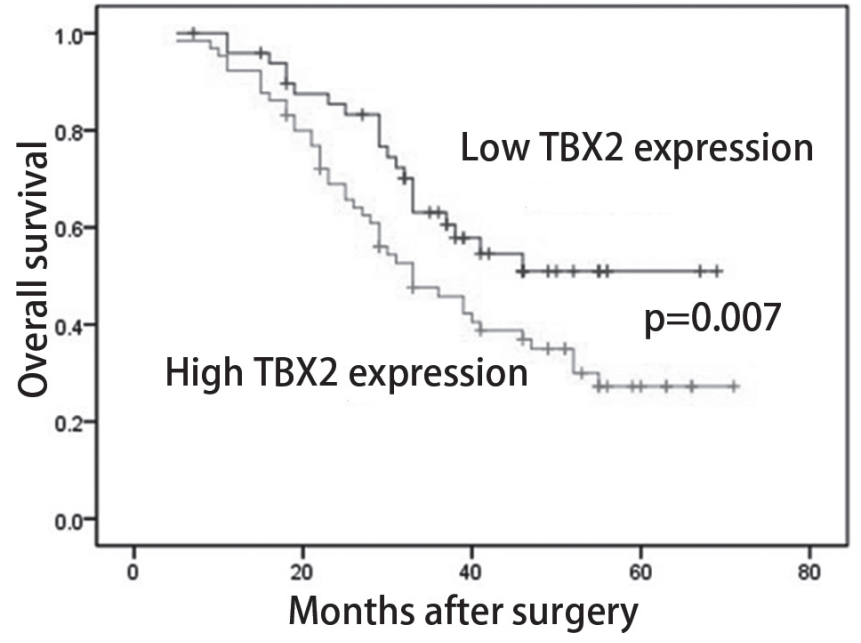

Figure 3. The Kaplan-Meier analysis of overall survival of non-small cell lung cancer (NSCLC) patients according to TBX2 expression status.

TBX2 is a member of the T-box family of transcription factors and has been implicated in several developmental processes, such as patterning and morphogenesis of a wide range of tissues and organs [11]. Moreover, TBX2 has also been involved in cell cycle regulation and carcinogenesis. Amplification of the chromosomal region 17q22-q24 was common in breast cancer and TBX2, located at 17q23, was shown to be overexpressed in breast cancer cell lines [7]. Meanwhile, data have also revealed that TBX2 potently downregulates the p19ARF tumor suppressor, thereby causing efficient immortalization of primary fibroblasts [8]. Moreover, researchers examined TBX2 expression levels in several human and mouse melanoma cell lines as well as primary human melanocytes by Western blot [9]. They found that TBX2 was overexpressed in all melanoma cell lines tested and plays an important role in maintaining proliferation and suppression of senescence in melanomas [9]. In addition, TBX2 was amplified in pancreatic cancer cell lines [10]. It has been reported that TBX2 is a specific genomic marker for late-stage ovarian cancers [12]. Similarly, TBX2 has also been recognized as prognostic markers in bladder cancer [13]. However, we know little about whether TBX2 was implicated in NSCLC.

In the present study, we elucidate the clinical significance of TBX2 in NSCLC. Our results indicated that upregulation of TBX2 might be associated with highly aggressive phenotype of NSCLC. TBX2 was a significant prognostic biomarker for NSCLC, independent of other clinicopathologic features. These results need to be verified in a larger, prospective, controlled, clinical study. In conclusion, our study evaluated the possibility of using TBX2 as a clinically relevant indicator for NSCLC patients. TBX2 might be valuable for evaluating prognosis. Hence, further studies on the mechanism by which TBX2 is involved in the development and progression of NSCLC are required.

\section{References}

[1] SUBRAMANIAM S, THAKUR RK, YADAV VK, NANDA R, CHOWDHURY S, et al. Lung cancer biomarkers: State of the art. J Carcinog 2013; 12: 3. http://dx.doi.org/10.4103/14773163.107958

[2] TSIM S, O`DOWD CA, MILROY R, DAVIDSON S. Staging of non-small cell lung cancer (NSCLC): a review. Respir Med 2010; 104: 1767-74. http://dx.doi.org/10.1016/ j.rmed.2010.08.005

[3] HILDEBRANDT MA, GU J, WU X. Pharmacogenomics of platinum-based chemotherapy in NSCLC. Expert Opin Drug Metab Toxicol 2009; 5: 745-55. http://dx.doi.org/10.1517/ 17425250902973711

[4] FILIPITS M, PIRKER R. Predictive markers in the adjuvant therapy of non-small cell lung cancer. Lung Cancer 2011; 74: 355-63. http://dx.doi.org/10.1016/j.lungcan.2011.06.005 
[5] KING M, ARNOLD JS, SHANSKE A, MORROW BE. T-genes and limb bud development. Am J Med Genet A. 2006; 140: 1407-13. http://dx.doi.org/10.1002/ajmg.a.31250

[6] ABRAHAMS A, PARKER MI, PRINCE S. The T-box transcription factor Tbx2: its role in development and possible implication in cancer. IUBMB Life. 2010; 62: 92-102.

[7] BARLUND M, MONNI O, KONONEN J, CORNELISON R, TORHORST J, et al. Multiple genes at 17q23 undergo amplification and overexpression in breast cancer. Cancer Res. 2000; 60: 5340-4.

[8] JACOBS JJ, KEBLUSEK P, ROBANUS-MAANDAG E, KRISTEL P, LINGBEEK M, et al. Senescence bypass screen identifies TBX2, which represses Cdkn2a (p19(ARF)) and is amplified in a subset of human breast cancers. Nat Genet. 2000; 26: 291-9. http://dx.doi.org/10.1038/81583

[9] VANCE KW, CARREIRA S, BROSCH G, GODING CR. Tbx2 is overexpressed and plays an important role in maintaining proliferation and suppression of senescence in melanomas. Cancer Res. 2005; 65: 2260-8. http://dx.doi.org/10.1158/00085472.CAN-04-3045
10] MAHLAMAKI EH, BARLUND M, TANNER M, GORUNOVA L, HOGLUND M, et al. Frequent amplification of $8 \mathrm{q} 24,11 \mathrm{q}, 17 \mathrm{q}$, and $20 \mathrm{q}$-specific genes in pancreatic cancer. Genes Chromosomes Cancer. 2002; 35: 353-8. http://dx.doi. org/10.1002/gcc.10122

11] LUSTIG KD, KROLL KL, SUN EE, KIRSCHNER MW. Expression cloning of a Xenopus T-related gene (Xombi) involved in mesodermal patterning and blastopore lip formation. Development. 1996; 122: 4001-12.

12] DIMOVA I, ORSETTI B, NEGRE V, ROUGE C, URSULE L, et al. Genomic markers for ovarian cancer at chromosomes 1,8 and 17 revealed by array CGH analysis. Tumori. 2009; 95: 357-66.

13] KANDIMALLA R, VAN TILBORG AA, KOMPIER LC, STUMPEL DJ, STAM RW, et al. Genome-wide analysis of CpG island methylation in bladder cancer identified TBX2, TBX3, GATA2, and ZIC4 as pTa-specific prognostic markers. Eur Urol. 2012; 61: 1245-56. http://dx.doi.org/10.1016/ j.eururo.2012.01.011 